\title{
Story-Based Context and Control for Robotic and Human Interactive Motion
}

Catie Cuan

Stanford University

Stanford, CA 94305 USA

ccuan@stanford.edu

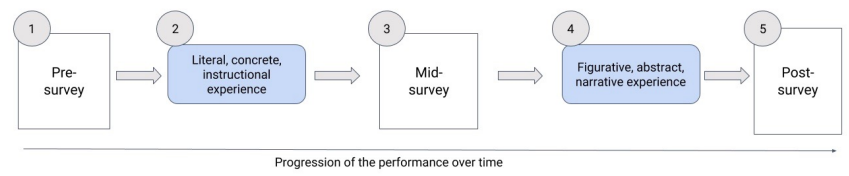

Figure 1: The embedding of a human-robot interaction experiment within a live performance.

Permission to make digital or hard copies of part or all of this work for personal or classroom use is granted without fee provided that copies are not made or distributed for profit or commercial advantage and that copies bear this notice and the full citation on the first page. Copyrights for third-party components of this work must be honored. For all other uses, contact the owner/author(s).

Copyright held by the owner/author(s)

CC' 19 , June 23-26, 2019, San Diego, CA, USA ACM 978-1-4503-5917-7/19/06.

https://doi.org/10.1145/3325480.3326558

\begin{abstract}
This extended abstract outlines the research plans and previous work of a first year PhD student applying to the Creativity and Cognition Graduate Student Symposium. The applicant's research interest is in designing and implementing motion-based interactions with robots, where the study participant is primed for the interactions through various narratives. Past work is described as well as an outline for future progress. The applicant is particularly interested in generating expressive motion for robots originating with human movers, captured by sensors and video.
\end{abstract}

\section{Author Keywords}

human-robot interaction; dance; movement; social robotics; narrative; priming

\section{Introduction}

In a 2014 Fast Company article, MIT robotics professor Cynthia Breazeal recounted seeing Star Wars as a 10 year old, "Your jaw just drops...In many ways those droids were full-fledged characters; they cared about people. That was what, I think, really sparked my imagination." The impact of stories like Star Wars on generations of eventual roboticists and non-roboticists alike is monumental. What if those stories could be activated in a research context, towards pinpointing how and why people perceive what they do about robots, and impact their behaviors as a result? 
Influential work in psychology has demonstrated that humans experience and conceive of their own lives through the context of story [15]. While increasing, the saturation of robots is relatively low when compared to other technologies like smart phones and televisions [16, 3]. Thus individuals' perceptions of robots may not be based on personal experience, but rather partially influenced by story-based methods like fiction and news.

In order to study existing perceptions and to generate new methods for interaction with robots, researchers have employed rich contexts, like theater and art installations [8, 9, $12,7,10]$. These contexts are an aesthetically and emotionally heightened test bed to experiment with robot expression.

Prior work has aimed to generate expressive robotic motion by assigning expressivity values and strategies [11]. Other work has demonstrated the impact of non-verbal, motionbased communication with robots on improved teamwork and efficiency [2]. Research has examined how a robot's movement and fictional backstory story can alter human behavior [6]. One method to generate expressive robotic motion is from video. OpenPose is a software library that estimates body, face, hand, and foot locations in real time, from multiple bodies in videos or pictures [13]. Robot Operating System is a robotics middleware that allows inputs from varying sources to create actions for a variety of robots in simulation and real life [14]. Prior work has created a ROS wrapper to generate $3 \mathrm{~d}$ pose estimation from a $2 \mathrm{~d}$ image combined with Kinect data [1].

These topics of investigation: how a robot's and human interactant's movement alters behavior in various contexts, the impact of stories in HRI, and generating robotic motion from moving human sources, are the foundations of my research. My work additionally employs theater and art in- stallations as experiment sites for human-robot interaction studies. My core research questions are:

- How do previous exposures to narratives or narrativebased experiments alter human behavior when interacting with robots?

- How can we advance sources of expressive motion for robots to make them easier to control and more acceptable by humans without programming backgrounds?

\section{Prior Work}

I utilize my background in choreography to design motionbased interactive experiments with robots. I then test those programs in narrative contexts (such as live performances, and art installations) to analyze how narrative can influence valence and empowerment when interacting non-verbally with robots. Conducting these experiments in a performance setting leads to a broad, diverse participant pool and is more similar to the rich performative contexts (like films and games) where participants may have been previously exposed to robots. I completed this initial work during my artist-in-residence period and research assistant affiliation with the Robotics, Automation, and Dance (RAD) Lab at the University of Illinois at Urbana-Champaign.

One development was CURTAIN [4], an experimental framework for human-robot interaction using a performance priming event followed by a designed series of dyadic, movementbased interactions with robots. This experiment framework is shown in Fig. 1 and Fig 2. The audience became a group of research participants in addition to being performance viewers. The audience completes three surveys which bookmark two experiences: a concrete, instructional experience, and a figurative, narrative experience. During the 


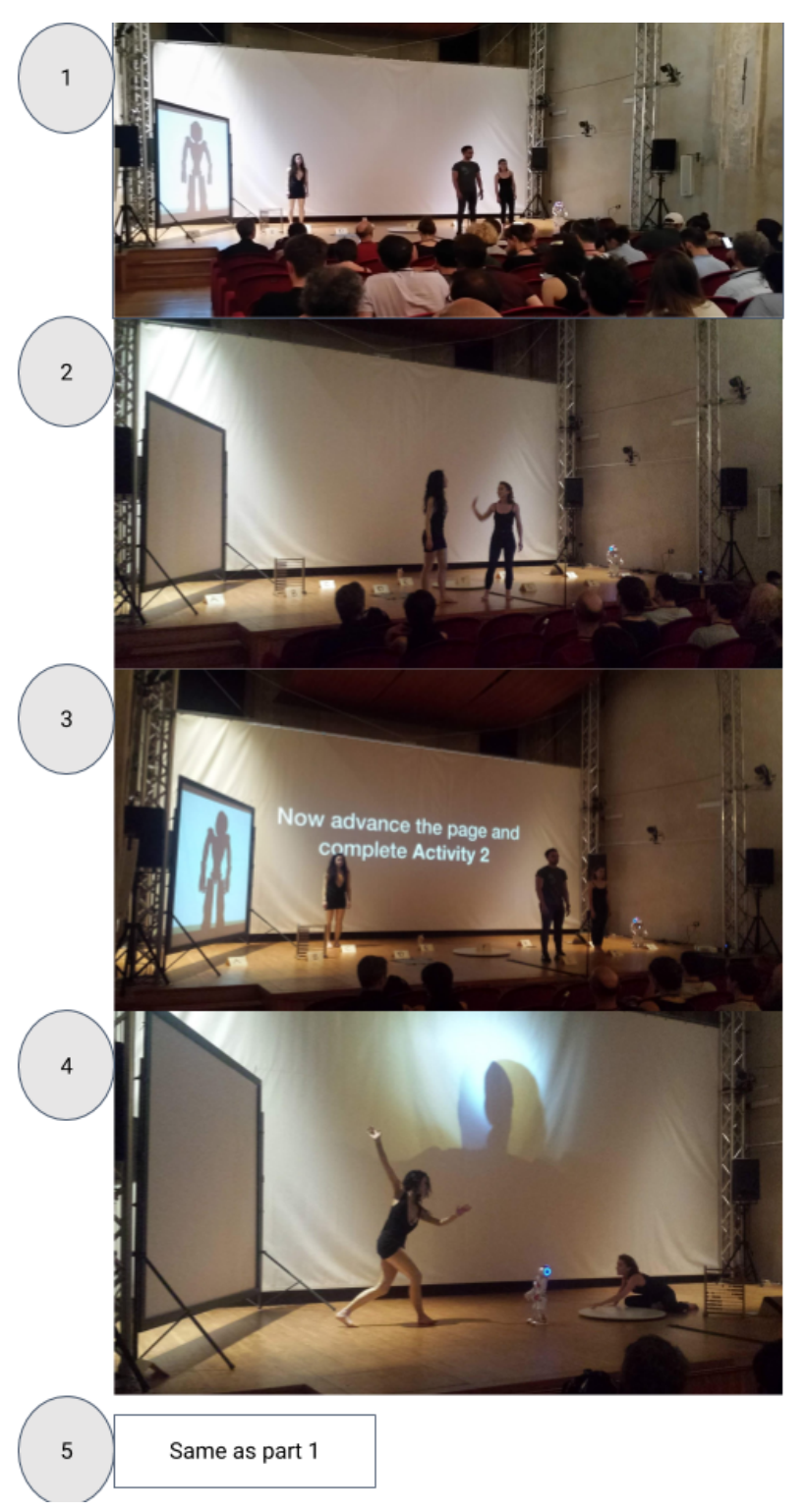

Figure 2: The CURTAIN experiment framework instituted with the Time to Compile performance. Performers and items are placed in a lineup during the survey portions for improved visibility. surveys, the audience is asked about the power, valence, acceptability, and expressivity of the various humans and items in the show. Included among these items are robots and theatrically-altered representations of robots. In this context, expressivity means the perceived complexity of the system in question.

Initial findings from this research demonstrate that technologies or humans which are included in performances are rated as more expressive; early findings support the hypothesis that humans overestimate expressivity in artificial systems and underestimate expressivity in natural systems. This trend is particularly evident across genders and individuals with differing programming experience. There is highest disagreement among groups when evaluating highly anthropomorphic agents.

A related body of research work designed a series of interactions without the performance priming, outlined in [5]. In this series of interactions, there are four partitioned spaces each equipped with a sensor and an actuator, including two robot actuators. Initial findings from this work show that humans have difficulty identifying the control mechanisms behind various human and robot agents, leading to further research questions about how perceptions of control affects perceptions of self-efficacy and self-assessment. In this body of work, robots are controlled expressively by moving humans detected by sensors like the Kinect and OptiTrack motion capture.

\section{Future Work}

I will extend the application of OpenPose and ROS to videos of moving dancers in order to diagnose motion patterns that will be the key templates for driving expressive robotic motion. I will then test this system in a performance context to see how motion and narrative-influenced methods of robotic 
control improves perceptions of robots and one's own technical capablities, particularly for participants with little or no programming background.

First, a set of moving dancer videos will be selected and tagged according to style and emotion (based on input from dance scholars and the Circumplex Model of Affect). An analysis of OpenPose on each tag set will begin the diagnosis of patterns in each type.

After this, points on the estimated video skeleton will be mapped onto different points of the robot. This will create a dynamic relationship between videos of expressively moving dancers and the motion of a robot. This software will then be oriented inside of an interactive art installation. For example, an installation would include a web camera, computer, and humanoid robot. Participants would be invited to enter the installation and move to control a robot. The participantâÁŹs movement would then be recorded by the web camera and saved into a video. The software program runs on the saved video and the robot will move expressively as a result. The research contribution is that an interface like this would allow individuals without programming backgrounds to control robots in a non-laboratory setting, possibly leading to increased empowerment when interacting with these typically inaccessible or intimidating machines. It would also provide information about the type of training or introduction scenarios that are optimal for new robots.

Research data will be collected at these public demonstrations, as participants share their perceptions of the robot before and after experiencing the installation, as well as their perceptions of their own technical capabilities, in pre and post-surveys (Semantic Differential Scales and my experience generating prior metrics to measuring robot perceptions inside art installations will be employed [5]).

\section{Anticipated Contributions for Broad Impact}

I have implemented various iterations of this performance and experiment design on four separate occasions. These public performances and experiments reach a broad group of individuals from a variety of backgrounds in different locations, thus bringing novel technologies like robots, virtual reality systems, and motion capture systems out of the lab and into communities. This work has the potential to increase comfort and empowerment for individuals without prior personal exposure to robots by exhibiting control over the device through an unusual mechanism like dance and a non-competitive setting like an art installation. This could inspire individuals, from typically underrepresented backgrounds, to study or work with robots in new ways.

A later impact is for robots to be able to perform these motion sequences in different contexts. For example, an application of this research work could have robots with recorded motion sequences (generated from participants in the installation and videos of moving dancers) that are available for selection by a human operator.

\section{Acknowledgements}

This work was conducted under IRB \#17427 and supported by NSF grant \#1528036 and DARPA award \#D16AP00001. The author wishes to thank Amy LaViers, Ishaan Pakrasi, Erin Berl, Andy McWilliams, Ellen Pearlman, and Cameron Scoggins for their contributions to this work.

\section{References}

[1] Miguel Arduengo García, Steven Jens Jorgensen, Kimberly Hambuchen, Luis Sentis, Francesc Moreno-Noguer, and Guillem Alenyà Ribas. 2017. ROS wrapper for real-time multi-person pose estimation with a single camera. (2017). 
[2] C. Breazeal, C.D. Kidd, A.L. Thomaz, G. Hoffman, and M. Berlin. 2005. Effects of nonverbal communication on efficiency and robustness in human-robot teamwork. In Intelligent Robots and Systems, 2005.(IROS 2005). 2005 IEEE/RSJ International Conference on. IEEE, 708-713.

[3] Pew Research Center. 2018. Mobile Fact Sheet. Washington: Pew Research Center (2018).

[4] C. Cuan, I. Pakrasi, E. Berl, and A. LaViers. 2018b. CURTAIN and Time to Compile: A Demonstration of an Experimental Testbed for Human-Robot Interaction. In RO-MAN, IEEE. IEEE, 255-261.

[5] Catie Cuan, Ishaan Pakrasi, and Amy LaViers. 2018a. Perception of Control in Artificial and Human Systems: A Study of Embodied Performance Interactions. In International Conference on Social Robotics. Springer, 503-512.

[6] Kate Darling, Palash Nandy, and Cynthia Breazeal. 2015. Empathic concern and the effect of stories in human-robot interaction. In Robot and Human Interactive Communication (RO-MAN), 2015 24th IEEE International Symposium on. IEEE, 770-775.

[7] Petra Gemeinboeck and Rob Saunders. 2015. Towards socializing non-anthropomorphic robots by harnessing dancers' kinesthetic awareness. In International Workshop in Cultural Robotics. Springer, 85-97.

[8] Ken Goldberg and Roland Siegwart. 2002. Beyond Webcams: an introduction to online robots. MIT press.

[9] Elizabeth Jochum, Evgenios Vlachos, Anja Christoffersen, Sally Grindsted Nielsen, Ibrahim A Hameed, and Zheng-Hua Tan. 2016. Using theatre to study interaction with care robots. International Journal of Social Robotics 8, 4 (2016), 457-470.

[10] Heather Knight. 2011. Eight lessons learned about non-verbal interactions through robot theater. In International Conference on Social Robotics. Springer, 42-51.

[11] Heather Knight and Reid Simmons. 2014. Expressive motion with $\mathrm{x}, \mathrm{y}$ and theta: Laban effort features for mobile robots. In The 23rd IEEE International Symposium on Robot and Human Interactive Communication. IEEE, 267-273.

[12] Chyi-Yeu Lin, Chang-Kuo Tseng, Wei-Chung Teng, Wei-Chen Lee, Chung-Hsien Kuo, Hung-Yan Gu, Kuo-Liang Chung, and Chin-Shyurng Fahn. 2009. The realization of robot theater: Humanoid robots and theatric performance. In 2009 International Conference on Advanced Robotics. IEEE, 1-6.

[13] Sen Qiao, Yilin Wang, and Jian Li. 2017. Real-time human gesture grading based on OpenPose. In 2017 10th International Congress on Image and Signal Processing, BioMedical Engineering and Informatics (CISP-BMEI). IEEE, 1-6.

[14] Morgan Quigley, Ken Conley, Brian Gerkey, Josh Faust, Tully Foote, Jeremy Leibs, Rob Wheeler, and Andrew Y Ng. 2009. ROS: an open-source Robot Operating System. In ICRA workshop on open source software, Vol. 3. Kobe, Japan, 5.

[15] Theordore R Sarbin. 1986. The narrative as a root metaphor for psychology. (1986).

[16] Nielsen National Television. 2017. Nielsen Estimates 119.6 Million TV Homes. Nielsen Insights (2017). 